\title{
A NOTE ON ELEMENTARY OPERATORS ON THE CALKIN ALGEBRA
}

\author{
JANKO GRAVNER
}

\begin{abstract}
Various parts of the spectrum of elementary operators on the Calkin algebra are characterised by means of the joint (Harte) spectra of $n$ tuples of operators.
\end{abstract}

1. Introduction and notation. Let $B$ be a complex Banach algebra with identity 1 . Then the left and right spectra of an $n$-tuple $\underline{a} \in B^{n}$ are the following subsets of $\mathbf{C}^{n}$ :

$$
\sigma_{1}(\underline{a})=\left\{\underline{s} \in \mathbf{C}^{n}, 1 \notin \sum_{j=1}^{n} B\left(a_{j}-s_{j}\right)\right\}
$$

and

$$
\sigma_{r}(\underline{a})=\left\{\underline{s} \in \mathbf{C}^{n}, 1 \notin \sum_{j=1}^{n}\left(a_{j}-s_{j}\right) B\right\},
$$

while the joint spectrum is the union

$$
\sigma_{j}(\underline{a})=\sigma_{1}(\underline{a}) \cup \sigma_{r}(\underline{a}) .
$$

We call an $n$-tuple $\underline{a} \in B^{n}$ commutative if $a_{j} a_{i}=a_{i} a_{j}$ for $i, j=1, \ldots, n$, and say that it commutes with an $m$-tuple $\underline{b} \in B^{m}$ if $a_{i} b_{j}=b_{j} a_{i}$ for each $i$ and $j$. If $\underline{a} \in B^{n}$ and $\underline{b} \in B^{m}$ are arbitrary tuples, and $\underline{g} \in$ Poly $_{m+n}^{p}$ is a $p$-tuple of polynomials in $n+m$ noncommuting variables, then the spectral mapping theorems of Harte can be summarised as follows [11, Theorem 1.2]: $B^{n+m}$

$(0.4) \underline{g} \sigma_{1}(\underline{a}, \underline{b}) \subset \sigma_{1}(\underline{g}(\underline{a}, \underline{b}))$ and $\underline{g} \sigma_{r}(\underline{a}, \underline{b}) \subset \sigma_{r}(\underline{g}(\underline{a}, \underline{b}))$ for arbitrary $(\underline{a}, \underline{b}) \in$

(0.5) $\sigma_{1}(\underline{g}(\underline{a}, \underline{b})) \subset \sigma_{1}\left(\underline{g}\left(\sigma_{1}(\underline{a}), \underline{b}\right)\right)$ and $\sigma_{r}(\underline{g}(\underline{a}, \underline{b})) \subset \sigma_{r}\left(\underline{g}\left(\sigma_{r}(\underline{a}), \underline{b}\right)\right)$ if $\underline{a} \in B^{n}$ commutes with $(\underline{a}, \underline{b}) \in B^{n+m}$;

(0.6) $\sigma_{1}(\underline{g}(\underline{a}, \underline{b}))=\underline{g} \sigma_{1}(\underline{a}, \underline{b})$ and $\sigma_{r}(\underline{g}(\underline{a}, \underline{b}))=\underline{g} \sigma_{r}(\underline{a}, \underline{b})$ if $(\underline{a}, \underline{b}) \in B^{n+m}$ is commutative. In particular, if $m=n$ we shall write

$$
\underline{a} \cdot \underline{b}=a_{1} b_{1}+\cdots+a_{n} b_{n} \in B
$$

and also define operators $L_{\underline{a}}: B \rightarrow B^{n}$ and $R_{\underline{b}}: B \rightarrow B^{n}$ by setting, for each $x \in B$,

$$
L_{\underline{a}}(x)=\underline{a} x=\left(a_{1} x, \ldots, a_{n} x\right) \quad \text { and } \quad R_{\underline{b}}(x)=x \underline{b}=\left(x b_{1}, \ldots, x b_{n}\right) .
$$

Received by the editors May 22, 1985.

1980 Mathematics Subject Classification. Primary 47C15, 47A62, 47A10.

Key words and phrases. Calkin algebra, elementary operator, joint spectra, von NeumannSchatten $p$-class, noncommutative Weyl-von Neumann theorem. 
In particular, in the algebra $\mathcal{L}(B)$ of all bounded operators on $B$,

$$
L_{\underline{a}} \cdot R_{\underline{b}}=R_{\underline{b}} \cdot L_{\underline{a}}: x \mapsto \underline{a} x \cdot \underline{b}=\underline{a} \cdot x \underline{b}=a_{1} x b_{1}+\cdots+a_{n} x b_{n}
$$

is the elementary operator on $\not$, induced by the $n$-tuples $\underline{a}$ and $\underline{b}$. Throughout this paper, $\not H$ will denote a fixed, infinite-dimensional Hilbert space. The Calkin algebra $\mathcal{L}(\mathcal{H} / \mathcal{K}(\mathcal{H})$ will be denoted by $\mathcal{A}$, while $\pi$ will be the quotient map from $\mathcal{L}(\mathcal{H})$ onto $A$. It is a purpose of this note to determine the spectrum of an elementary operator $L_{\underline{a}} \cdot R_{\underline{b}}$ when $\underline{a} \in A^{n}$ and $\underline{b} \in \mathcal{A}^{n}$ and either $\underline{a}$ or $\underline{b}$ is commutative.

Spectral properties of elementary operators on $\mathcal{L}(\mathcal{H})$ have been discussed by Harte [11, Theorem 3.5]: If $B=\mathcal{L}(\not{H})$ and $\underline{a} \in B^{n}$ is commutative, then

$$
\begin{aligned}
& \sigma_{1}\left(L_{\underline{a}} \cdot R_{\underline{b}}\right)=\sigma_{r}\left(\sigma_{1}(\underline{a}) \cdot \underline{b}\right)=\bigcup\left\{\sigma_{r}(\underline{s} \cdot \underline{b}), \underline{s} \in \sigma_{1}(\underline{a})\right\}, \\
& \sigma_{r}\left(L_{\underline{a}} \cdot R_{\underline{b}}\right)=\sigma_{1}\left(\sigma_{r}(\underline{a}) \cdot \underline{b}\right)=\bigcup\left\{\sigma_{1}(\underline{s} \cdot \underline{b}), \underline{s} \in \sigma_{r}(\underline{a})\right\} .
\end{aligned}
$$

Our first result shows that $(0.10)$ and $(0.11)$ continue to hold when $\mathcal{L}(\mathcal{H})$ is replaced by $A$, and we shall also show that the left and the right spectra of the restriction of $L_{\underline{a}} \cdot R_{\underline{b}}$ to the von Neumann-Schatten $p$-class $C_{p}(\not H) \subset \mathcal{L}(\not H)$ are the same as the spectrum of $L_{\underline{a}} \cdot R_{\underline{b}}$ on $\mathcal{L}(\mathscr{H})$. We should remark that both these situations have been studied by Fialkow (see [4-6], which include some further references) and Carillo and Hernandez [2] under the more restrictive condition that $\underline{a}$ and $\underline{b}$ are both commutative. Some special cases of our results can also be found in [15].

We recall that approximate point and defect spectrum of an operator $T$ acting on a Banach space are, respectively, the sets

$$
\sigma_{\pi}(T)=\{\lambda \in \mathbf{C}, T-\lambda \text { is not bounded below }\}
$$

and

$$
\sigma_{\delta}(T)=\{\lambda \in \mathbf{C}, T-\lambda \text { is not surjective }\} .
$$

We will also prove that for elementary operators on $\mathcal{L}(\mathcal{H})$ and on $\mathcal{A}$, the left spectrum is equal to the approximate point spectrum and the right spectrum is equal to the defect spectrum.

1. The spectra. . We begin with a preliminary result, originally due to Wolf, which is given by Dash [3] and by Fillmore, Stampfli and Williams [7]. then

LEMMA 1. If $\underline{a} \in \mathcal{A}^{n}$ is a commutative $n$-tuple of elements in the Calkin algebra,

(1.1) $\underline{s} \in \sigma_{1}(\underline{a})$ if and only if $(\underline{a}-\underline{s}) \pi(P)=0$ for a noncompact projection $P \in \mathcal{L}(\mathcal{H})$, and

(1.2) $\underline{s} \in \sigma_{r}(\underline{a})$ if and only if $\pi(P)(\underline{a}-\underline{s})=0$ for a noncompact projection $P \in \mathcal{L}(\mathcal{H})$. In particular, the left and the right spectra of a consist, respectively, of the left point spectrum and the right point spectrum.

THEOREM 1. Let $L_{\underline{a}} \cdot R_{\underline{b}}$ be an elementary operator on $\mathcal{A}$. If $\underline{a} \in A^{n}$ is commutative, then the following spectral equalities are valid:

$$
\sigma_{1}\left(L_{\underline{a}} \cdot R_{\underline{b}}\right)=\sigma_{r}\left(\sigma_{1}(\underline{a}) \cdot \underline{b}\right)
$$

and

$$
\sigma_{r}\left(L_{\underline{a}} \cdot R_{\underline{b}}\right)=\sigma_{1}\left(\sigma_{r}(\underline{a}) \cdot \underline{b}\right)
$$


and if $\underline{b}$ is commutative, then

$$
\sigma_{1}\left(L_{\underline{a}} \cdot R_{\underline{b}}\right)=\sigma_{1}\left(\sigma_{r}(\underline{b}) \cdot \underline{a}\right)
$$

and

$$
\sigma_{r}\left(L_{\underline{a}} \cdot R_{\underline{b}}\right)=\sigma_{r}\left(\sigma_{1}(\underline{b}) \cdot \underline{a}\right) .
$$

Further, in both cases $\sigma_{1}\left(L_{\underline{a}} \cdot R_{\underline{b}}\right)$ consists of eigenvalues $\lambda \in \sigma_{p}\left(L_{\underline{a}} \cdot R_{\underline{b}}\right)$ with infinite-dimensional $\operatorname{Ker}\left(L_{\underline{a}} \cdot R_{\underline{b}}-\lambda\right)$, and for each $\lambda \in \sigma_{r}\left(L_{\underline{a}} \cdot R_{\underline{b}}\right)$,

$$
\operatorname{codim} \operatorname{Im}\left(L_{\underline{a}} \cdot R_{\underline{b}}-\lambda\right)^{-}=\operatorname{dim} A / \operatorname{Im}\left(L_{\underline{a}} \cdot R_{\underline{b}}-\lambda\right)^{-}=\infty \text {. }
$$

PROOF. We will restrict ourselves to the case of commutative $\underline{a}$; the case of commutative $\underline{b}$ then follows by taking adjoints. The two inclusions $\sigma_{1}\left(L_{\underline{a}} \cdot R_{\underline{b}}\right) \subset$ $\sigma_{r}\left(\sigma_{1}(\underline{a}) \cdot \underline{b}\right)$ and $\sigma_{r}\left(L_{\underline{a}} \cdot R_{\underline{b}}\right) \subset \sigma_{1}\left(\sigma_{r}(\underline{a}) \cdot \underline{b}\right)$ follow from $(0.5)$.

We can clearly assume that the range of $P$, obtained from Lemma 1 , is separable. Throughout this proof, $P$ and $Q$ will be projections with separable infinitedimensional ranges, $\left\{\mathcal{M}_{i}, i=1,2, \ldots\right\}$ will be an orthogonal family of infinitedimensional closed subspaces of $\operatorname{Im} P$, and $U_{i}$ are partial isometries with initial spaces $\operatorname{Im} Q$ and final spaces $M_{i}$.

Take $\underline{s} \in \sigma_{1}(\underline{a})$ and $\lambda \in \sigma_{r}(\underline{s} \cdot \underline{b})$ By the previous lemma, there exist $P$ and $Q$ such that $(\underline{a}-\underline{s}) \pi(P)=0$ and $\pi(Q)(\underline{s} \cdot \underline{b}-\lambda)=0$. It is easy to see that the elements $\pi\left(P U_{i} Q\right)$ are linearly independent in $\mathcal{A}$. But

$$
\left(L_{\underline{a}} \cdot R_{\underline{b}}-\lambda\right) \pi\left(P U_{i} Q\right)=(\underline{a}-\underline{s}) \pi\left(P U_{i} Q\right) \cdot \underline{b}+\pi\left(P U_{i} Q\right)(\underline{s} \cdot \underline{b}-\lambda)=0,
$$

proving the assertions about the left spectrum.

Now, take $\underline{s} \in \sigma_{r}(\underline{a})$ and $\lambda \in \sigma_{1}(\underline{s} \cdot \underline{b})$ and choose $P$ and $Q$ such that $\pi(P)(\underline{a}-\underline{s})=$ 0 and $(\underline{s} \cdot \underline{b}-\lambda) \pi(Q)=0$. For every $x \in A$ denote by $[x]$ the coset of $x$ in $A / \operatorname{Im}\left(L_{\underline{a}} \cdot R_{\underline{b}}-\lambda\right)^{-}$. Choose any scalars $t_{1}, \ldots, t_{m}$ and assume that

$$
\sum_{j=1}^{m}\left[t_{j} \pi\left(P U_{j} Q\right)\right]=0
$$

In this case

$$
\begin{aligned}
0 & =\left\|\left[\sum t_{j} \pi\left(P U_{j} Q\right)\right]\right\| \\
& =\inf _{x \in \mathcal{A}}\left\|\underline{a} x \cdot \underline{b}-\lambda x-\sum t_{j} \pi\left(P U_{j} Q\right)\right\| \\
& \geq \inf _{x \in \mathcal{A}}\left\|\pi(P) \underline{a} x \cdot \underline{b} \pi(Q)-\lambda \pi(P) x \pi(Q)-\sum t_{j} \pi\left(P U_{j} Q\right)\right\| \\
& =\inf _{x \in \mathcal{A}}\left\|\pi(P)(\underline{a}-\underline{s}) x \cdot \underline{b} \pi(Q)+\pi(P) x(\underline{s} \cdot \underline{b}-\lambda) \pi(Q)-\sum t_{j} \pi\left(P U_{j} Q\right)\right\| \\
& =\left\|\sum t_{j} \pi\left(P U_{j} Q\right)\right\|,
\end{aligned}
$$

showing that $\sum_{j=1}^{m} t_{j} \pi\left(P U_{j} Q\right)=0$. Since $\pi\left(P U_{j} Q\right)$ are linearly independent, it follows that $t_{1}=\cdots=t_{m}=0$. The cosets $\left[\pi\left(P U_{j} Q\right)\right]$ are therefore linearly independent and $\operatorname{codim} \operatorname{Im}\left(L_{\underline{a}} \cdot R_{\underline{b}}-\lambda\right)^{-}=\infty$. 
COROLLARY 1. An elementary operator $L_{\underline{a}} \cdot R_{\underline{b}}$ on $A$ with $\underline{a}$ commutative is semi-Fredholm if and only if it is invertible from one side, and Fredholm if and only if it is invertible; thus $\sigma_{e}\left(L_{\underline{a}} \cdot R_{\underline{b}}\right)=\sigma\left(L_{\underline{a}} \cdot R_{\underline{b}}\right)$.

Theorem 1 already gives a formula for the spectrum of an elementary operator on the Calkin algebra. But we wish to prove another, more elegant, formula. To this end we have to clarify the situation for elementary operators on $\mathcal{L}(\mathcal{H})$ first. In our proof of the next theorem, we use techniques very similar to those in $[\mathbf{1 1}]$ and [12], so we will be brief.

Let $L_{\underline{a}} \cdot R_{\underline{b}}$ be an elementary operator on $\mathcal{L}(\mathcal{H})$. By $L_{\underline{a}}^{p}$ we denote the $n$-tuple of restrictions $L_{a_{i}} \mid C_{p}(\mathscr{H}), i=1, \ldots, n$, and $R_{b}^{p}$ has an analogous meaning. Thus $L_{\underline{a}}^{p} \cdot R_{\underline{b}}^{p}$ is the restriction of $L_{\underline{a}} \cdot R_{\underline{b}}$ to the von Neumann-Schatten $p$-class $C_{p}(\not H)$.

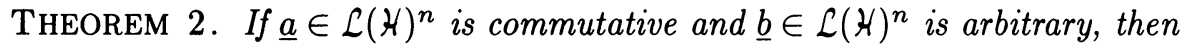

$$
\begin{aligned}
& \sigma_{1}\left(L_{\underline{a}}^{p} \cdot R_{\underline{b}}^{p}\right)=\sigma_{1}\left(L_{\underline{a}} \cdot R_{\underline{b}}\right)=\sigma_{\pi}\left(L_{\underline{a}} \cdot R_{\underline{b}}\right)=\sigma_{r}\left(\sigma_{1}(\underline{a}) \cdot \underline{b}\right), \\
& \sigma_{r}\left(L_{\underline{a}}^{p} \cdot R_{\underline{b}}^{p}\right)=\sigma_{r}\left(L_{\underline{a}} \cdot R_{\underline{b}}\right)=\sigma_{\delta}\left(L_{\underline{a}} \cdot R_{\underline{b}}\right)=\sigma_{1}\left(\sigma_{r}(\underline{a}) \cdot \underline{b}\right)
\end{aligned}
$$

and

$$
\sigma\left(L_{\underline{a}}^{p} \cdot R_{\underline{b}}^{p}\right)=\sigma\left(L_{\underline{a}} \cdot R_{\underline{b}}\right)=\sigma\left(\sigma_{j}(\underline{a}) \cdot \underline{b}\right)=\bigcup\left\{\sigma(\underline{s} \cdot \underline{b}), \underline{s} \in \sigma_{j}(\underline{a})\right\} .
$$

If, instead, $\underline{b} \in \mathcal{L}(\mathcal{K})^{n}$ is commutative, then

$$
\begin{aligned}
& \sigma_{1}\left(L_{\underline{a}}^{p} \cdot R_{\underline{b}}^{p}\right)=\sigma_{1}\left(L_{\underline{a}} \cdot R_{\underline{b}}\right)=\sigma_{\pi}\left(L_{\underline{a}} \cdot R_{\underline{b}}\right)=\sigma_{1}\left(\sigma_{r}(\underline{b}) \cdot \underline{a}\right), \\
& \sigma_{r}\left(L_{\underline{a}}^{p} \cdot R_{\underline{b}}^{p}\right)=\sigma_{r}\left(L_{\underline{a}} \cdot R_{\underline{b}}\right)=\sigma_{\delta}\left(L_{\underline{a}} \cdot R_{\underline{b}}\right)=\sigma_{r}\left(\sigma_{1}(\underline{b}) \cdot \underline{a}\right)
\end{aligned}
$$

and

$$
\sigma\left(L_{\underline{a}}^{p} \cdot R_{\underline{b}}^{p}\right)=\sigma\left(L_{\underline{a}} \cdot R_{\underline{b}}\right)=\sigma\left(\sigma_{j}(\underline{b}) \cdot a\right) .
$$

PROOF. If $\underline{a}$ is commutative, the inclusions

$$
\sigma_{1}\left(L_{\underline{a}} \cdot R_{\underline{b}}\right) \subset \sigma_{r}\left(\sigma_{1}(\underline{a}) \cdot \underline{b}\right) \quad \text { and } \quad \sigma_{r}\left(L_{\underline{a}} \cdot R_{\underline{b}}\right) \subset \sigma_{1}\left(\sigma_{r}(\underline{a}) \cdot \underline{b}\right)
$$

again follow from (0.5). To prove that $\sigma_{r}\left(\sigma_{1}(\underline{a}) \cdot \underline{b}\right) \subset \sigma_{\pi}\left(L_{\underline{a}} \cdot R_{\underline{b}}\right)$, take an $\underline{s} \in \sigma_{1}(\underline{a})$ and a $\lambda \in \sigma_{r}(\underline{s} \cdot \underline{a})$. Then there exist sequences $\left(x_{k}\right)$ and $\left(y_{k}\right)$ of unit vectors such that $\left(a_{i}-s_{i}\right) x_{k} \rightarrow_{k} 0$, for $i=1, \ldots, n$ and $(\underline{s} \cdot \underline{a}-\lambda) y_{k} \rightarrow_{k} 0$. The operators $Z_{k}=x_{k} \otimes y_{k}$ have norm 1 , and it is easy to see that $\left(L_{\underline{a}} \cdot R_{\underline{b}}-\lambda\right) Z_{k} \rightarrow_{k} 0$. Thus $\lambda \in \sigma_{\pi}\left(L_{\underline{a}} \cdot R_{\underline{b}}\right)$.

The same argument, of course, works for an operator $L_{\underline{a}}^{p} \cdot R_{\underline{b}}^{p}$. Hence (1.7) holds. We omit the proof of (1.10).

The assertions concerning the right spectra, (1.8) and (1.11), can now be proved by exploiting the following connections between the operators $L_{\underline{a}}^{p} \cdot R_{\underline{b}}^{p}$ and their Banach space adjoints: $\left(L_{\underline{a}}^{p} \cdot R_{\underline{b}}^{p}\right)^{\prime}=\left(L_{\underline{b}}^{q} \cdot R_{\underline{a}}^{q}\right)$, where $1 / p+1 / q=1$ and $1 \leq p<\infty$ (if we set $\left.C_{\infty}(\not H)=K(\not H)\right)$ and $\left(L_{\underline{b}}^{1} \cdot R_{\underline{a}}^{\overline{1}}\right)^{\prime}=L_{\underline{a}} \cdot R_{\underline{b}}$. To prove (1.9) and (1.11), we will first see that if $\underline{a}$ is commutative then $\sigma_{1}\left(\sigma_{1}(\underline{a}) \cdot \underline{b}\right) \subset \sigma\left(L_{\underline{a}} \cdot R_{\underline{b}}\right)$. To this end, take an $\underline{s} \in \sigma_{1}(\underline{a})$ and a $\lambda \in \sigma_{1}(\underline{s} \cdot \underline{a})$. In this case we can choose sequences $\left(x_{k}\right)$ and $\left(y_{k}\right)$ of unit vectors such that $\left(a_{i}-s_{j}\right) x_{k} \rightarrow_{k} 0$ for $i=1, \ldots, n$ and $(\underline{s} \cdot \underline{b}-\lambda) y_{k} \rightarrow_{k} 0$. Define operators $X_{k}=x_{k} \otimes x_{k}, Y_{k}=y_{k} \otimes y_{k}$ and $Z_{k}=x_{k} \otimes y_{k}$. Assume that 
$L_{\underline{a}} \cdot R_{\underline{b}}-\lambda$ is invertible. Then there exists a bounded sequence of operators $\left(U_{k}\right)$ such that $\left(L_{\underline{a}} \cdot R_{\underline{b}}-\lambda\right) U_{k}=X_{k} Z_{k}$. Because the $n$-tuple $\underline{a}$ is commutative,

$$
\left(L_{\underline{a}} \cdot R_{\underline{b}}-\lambda\right)\left(\left(a_{i}-s_{i}\right) U_{k}\right)=\left(a_{i}-s_{i}\right)\left(L_{\underline{a}} \cdot R_{\underline{b}}-\lambda\right) U_{k}=\left(a_{i}-s_{i}\right) X_{k} Z_{k} \underset{k}{\rightarrow} 0
$$

for every $i$. Hence $\left(a_{i}-s_{i}\right) U_{k} \rightarrow{ }_{k} 0$ for every $i$, and

$$
\begin{aligned}
1 & =\left\|X_{k} Z_{k} Y_{k}\right\|=\left\|\left(\left(L_{\underline{a}} \cdot R_{\underline{b}}-\lambda\right) U_{k}\right) Y_{k}\right\| \\
& \leq \sum_{i=1}^{n}\left\|\left(a_{i}-s_{i}\right) U_{k}\right\|\left\|Y_{k}\right\|\left\|b_{i}\right\|+\left\|U_{k}\right\|\left\|(\underline{s} \cdot \underline{b}-\lambda) Y_{k}\right\|,
\end{aligned}
$$

but this tends to zero as $k$ increases, a contradiction. An analogous argument shows that $\sigma_{r}\left(\sigma_{r}(\underline{b}) \cdot \underline{a}\right) \subset \sigma\left(L_{\underline{a}} \cdot R_{\underline{b}}\right)$ in the case of commutative $\underline{b}$. Again both inclusions remain valid if we replace $L_{\underline{a}} \cdot R_{\underline{b}}$ by $L_{\underline{a}}^{p} \cdot R_{\underline{b}}^{p}$, where $p$ is arbitrary.

Now if again $\underline{a}$ is commutative, $\underline{s} \in \sigma_{r}(\underline{a})$ and $\lambda \in \sigma_{r}(\underline{s} \cdot \underline{b})$, then, by the previous discussion (with the roles of $\underline{a}$ and $\underline{b}$ interchanged), $\lambda \in \sigma\left(L_{\underline{b}}^{1} \cdot R_{\underline{a}}^{1}\right.$. But $\sigma\left(L_{\underline{b}}^{1} \cdot R_{\underline{a}}^{1}\right)=$ $\sigma\left(\left(L_{\underline{b}}^{1} \cdot R_{\underline{a}}^{1}\right)^{\prime}\right)=\sigma\left(L_{\underline{a}} \cdot R_{\underline{b}}\right)$. Thus $\lambda \in \sigma\left(L_{\underline{a}} \cdot R_{\underline{b}}\right)$ and $\sigma_{r}\left(\sigma_{r}(\underline{a}) \cdot \underline{b}\right) \subset \sigma\left(L_{\underline{a}} \cdot R_{\underline{b}}\right)$. A similar duality argument proves the same for the operators $L_{\underline{a}}^{p} \cdot R_{\underline{b}}^{p}$ and concludes the proof of (1.9). We again omit the proof for commutative $\underline{b}$.

In the sequel we will use the well-known noncommutative Weyl-von Neumann theorem of Voiculescu [14, Theorem 1.3]. We recall that two representations $\rho$ and $\tau$ of separable $C^{*}$-algebra $B$ on separable Hilbert spaces $\mathcal{H}_{\rho}$ and $\varkappa_{\tau}$ are said to be approximately equivalent $(\rho \stackrel{\mathrm{a}}{\sim} \tau)$ if there exists a sequence $U_{n}: \mathcal{H}_{\rho} \rightarrow \mathcal{H}_{\tau}$ of unitary operators such that, for each $A$ in $B$,

(1.13) $U_{n} \rho(A) U_{n}^{*}-\tau(A)$ are compact operators for every $n$ and their norms tend to zero as $n$ increases.

An equivalent formulation of Voiculescu's theorem (see [1, p. 339]) is then stated in the following lemma.

LEMMA 2. Let $B$ be a separable $C^{*}$-algebra of operators on a separable Hilbert space, $\rho$ a nondegenerate representation of $B$ on a separable Hilbert space which annihilates all compact operators from $B$, and id the identity representation of $B$. Then id $\oplus \rho \stackrel{\text { a }}{\sim}$ id.

Henceforth we will assume that if $a_{i}$ and $b_{i}$ are elements in the Calkin algebra $A$ then $A_{i}$ and $B_{i}$ are operators on $\forall$ for which $\pi\left(A_{i}\right)=a_{i}$ and $\pi\left(B_{i}\right)=b_{i}$ or, in short, $\pi(\underline{A})=\underline{a}$ and $\pi(\underline{B})=\underline{b}$.

The argument in the proof of the following proposition is partly similar to that in $[8$, Theorem 3].

Proposition. Assume the Hilbert space $\sharp$ to be separable. Take arbitrary $n$-tuples $\underline{a}, \underline{b} \in A^{n}$. Let $B$ be an arbitrary separable $C^{*}$-subalgebra $\mathcal{L}(\mathcal{H})$, which includes operators $A_{1}, \ldots, A_{n}$ and $B_{1}, \ldots, B_{n}$ and the identity $I$. Let $\rho$ be a faithful representation of the separable $C^{*}$-algebra $\pi(B) \subset A$ on a separable Hilbert space $\mathscr{H}_{\rho}$. Denote by $\mathcal{E}$ the elementary operator on $\mathcal{L}\left(\mathcal{H}_{\rho}\right)$ which maps $Z \in \mathcal{L}\left(\mathcal{H}_{\rho}\right)$ into $\sum_{i=1}^{n} \rho \pi\left(A_{i}\right) Z \rho \pi\left(B_{i}\right)$. Then

(1.14) If $L_{\underline{a}} \cdot R_{\underline{b}}$ is bounded below, then $\mathcal{E}$ is bounded below.

(1.15) If $L_{\underline{a}} \cdot R_{\underline{b}}$ is onto, then $\operatorname{Im} \mathcal{E}$ is dense in $\mathcal{L}\left(\mathcal{H}_{\rho}\right)$.

(1.16) If $L_{\underline{a}} \cdot R_{\underline{b}}$ is invertible, then $\mathcal{E}$ is invertible. 
PROOF. Let the representation $\rho^{\infty}$ be the direct sum of countably many copies of $\rho$. Then $\rho^{\infty}$ maps into $\mathcal{L}\left(\mathcal{H}_{\rho}^{\infty}\right), \mathfrak{H}_{\rho}^{\infty}$ being the direct sum of infinitely many copies of $\mathcal{H}_{\rho}$. If $T \in \mathcal{L}\left(\mathcal{H}_{\rho}\right)$, of course $T^{\infty} \in \mathcal{L}\left(\mathcal{H}_{\rho}^{\infty}\right)$ denotes the infinite direct sum $T \oplus T \oplus \cdots$. By the previous lemma id $\stackrel{\text { a }}{\sim}$ id $\oplus \rho^{\infty}$. In particular, there exists a unitary operator $U: \not{H} \oplus \mathcal{H}_{\rho}^{\infty} \rightarrow \not{H}$ such that $U^{*} A_{i} U-A_{i} \oplus \rho^{\infty} \pi\left(A_{i}\right)$ and $U^{*} B_{i} U-B_{i} \oplus \rho^{\infty} \pi\left(B_{i}\right)$ are compact for $i=1, \ldots, n$.

Assume first that $L_{\underline{a}} \cdot R_{b}$ is bounded below. Thus, if $\left(X_{k}\right)$ is a sequence of operators on $\mathcal{H}$ such that $\pi\left(\underline{A} X_{k} \cdot \underline{B}\right) \rightarrow_{k} 0$, then $\pi\left(X_{k}\right) \rightarrow_{k} 0$. Choose a sequence $\left(Z_{k}\right)$ of operators on $\mathscr{H}_{\rho}$ for which $\mathcal{E} Z_{k} \rightarrow_{k} 0$. Then clearly $0 \oplus\left(\mathcal{E} Z_{k}\right)^{\infty} \rightarrow_{k} 0$. But

$$
\begin{aligned}
0 \oplus\left(\mathcal{E} Z_{k}\right)^{\infty} & =\sum_{i=1}^{n}\left(A_{i} \oplus \rho^{\infty} \pi\left(A_{i}\right)\right)\left(0 \oplus Z_{k}^{\infty}\right)\left(B_{i} \oplus \rho^{\infty} \pi\left(B_{i}\right)\right) \\
& =\sum_{i=1}^{n} U^{*} A_{i} U\left(0 \oplus Z_{k}^{\infty}\right) U^{*} B_{i} U+K_{k},
\end{aligned}
$$

where $K_{k}$ is a compact operator on $\mathscr{H}$, which depends on $Z_{k}$. Thus

$$
\pi\left(\underline{a} \pi\left(U\left(0 \oplus Z_{k}^{\infty}\right) U^{*}\right) \cdot \underline{b}\right) \underset{k}{\rightarrow} 0 .
$$

From the fact that $T \mapsto \pi\left(U\left(0 \oplus T^{\infty}\right) U^{*}\right)$ is an injective $*$-homomorphism from $\mathcal{L}\left(\mathcal{H}_{\rho}\right)$ into $\mathcal{A}$, it follows that $\left\|\pi\left(U\left(0 \oplus Z_{k}^{\infty}\right) U^{*}\right)\right\|=\left\|Z_{k}\right\|$. From this and from the assumed boundedness below of $L_{\underline{a}} \cdot R_{\underline{b}}$, it follows that $Z_{k} \rightarrow{ }_{k} 0$. Hence $\mathcal{E}$ is bounded below.

To prove (1.15) assume that $L_{a} \cdot R_{b}$ is surjective, which means that for every $Y \in \mathcal{L}(\mathcal{H})$ there exists an $X \in \mathcal{L}(\mathcal{H})$ such that $\underline{A} X \cdot \underline{B}-Y \in \mathcal{K}(\mathcal{H})$. Thus, for every operator $W \in \mathcal{L}\left(\mathcal{H} \oplus \mathcal{H}_{\rho}^{\infty}\right)$ there exists a $Z \in \mathcal{L}\left(\mathcal{H} \oplus \mathcal{H}_{\rho}^{\infty}\right)$ such that

$$
\sum_{i=1}^{n}\left(A_{i} \oplus \rho^{\infty} \pi\left(A_{i}\right)\right) Z\left(B_{i} \oplus \rho^{\infty} \pi\left(B_{i}\right)\right)-W \in \mathcal{K}\left(\mathcal{H} \oplus \mathcal{H}_{\rho}^{\infty}\right) .
$$

Now take arbitrary $T \in \mathcal{L}\left(\mathcal{H}_{\rho}\right)$ and let $W$ be of the form $0 \oplus T^{\infty}$. Let $P_{k} \in \mathcal{L}\left(\mathcal{H}^{\prime} \oplus \mathcal{H}_{\rho}^{\infty}\right)$ be the projection on the $k$ th coordinate space in $\nVdash_{\rho}^{\infty}$. Then it is easy to see that the operators

$$
\sum_{i=1}^{n} \rho \pi\left(A_{i}\right)\left(P_{k} Z \mid \operatorname{Im} P_{k}\right) \rho \pi\left(B_{i}\right)-T
$$

are compact for every $k=1,2, \ldots$ and their norms tend to zero as $k$ tends to infinity. Thus $\operatorname{Im} \mathcal{E}$ is dense in $\mathcal{L}\left(\mathcal{H}_{\rho}\right)$, and the proposition is proved.

THEOREM 3. If $\underline{a}, \underline{b} \in \mathscr{A}^{n}$ and $\underline{a}$ is commutative, then

$$
\sigma\left(L_{\underline{a}} \cdot R_{\underline{b}}\right)=\sigma\left(\sigma_{j}(\underline{a}) \cdot \underline{b}\right) ;
$$

and if $\underline{b}$ is commutative, then

$$
\sigma\left(L_{\underline{a}} \cdot R_{\underline{b}}\right)=\sigma\left(\sigma_{j}(\underline{b}) \cdot \underline{a}\right) .
$$

ProOF. Again, it suffices to prove (1.17). In this case, by Theorem 1, only the inclusions

$$
\sigma_{1}\left(\sigma_{1}(\underline{a}) \cdot \underline{b}\right) \subset \sigma\left(L_{\underline{a}} \cdot R_{\underline{b}}\right), \quad \sigma_{r}\left(\sigma_{r}(\underline{a}) \cdot \underline{b}\right) \subset \sigma\left(L_{\underline{a}} \cdot R_{\underline{b}}\right)
$$

remain to be proved. 
The proof of (1.19) for separable $\not$ follows from the Proposition. For if $\underline{s} \in \sigma_{1}(\underline{a})$ and $\lambda \in \sigma_{1}(\underline{s} \cdot \underline{b})$, then we take some noncompact projections $P$ and $Q$ such that $(\underline{a}-\underline{s}) \pi(P)=0$ and $(\underline{s} \cdot \underline{b}-\lambda) \pi(Q)=0$, and let $B$ be the $C^{*}$-algebra generated by $A_{1}, \ldots, A_{n}, B_{1}, \ldots, B_{n}, I, P$ and $Q$, and $\rho$ an injective representation of $\pi(B)$. Then $(\rho(\underline{a})-\underline{s}) \rho \pi(P)=0$ and $\underline{s} \in \sigma_{1}(\rho(\underline{a}))$ since $\rho \pi(P) \neq 0$. Similarly $\lambda \in \sigma_{1}(\rho(\underline{s} \cdot \underline{b}))$. By Theorem $2, \mathcal{E}-\lambda$ cannot be invertible, and by the previous proposition neither can $L_{\underline{a}} \cdot R_{\underline{b}}-\lambda$. This proves the first inclusion in (1.19); an analogous argument proves the second.

If $H$ is not separable, then first note that there exists an infinite-dimensional separable Hilbert space $\mathscr{H}_{0}$, which reduces all $A_{i}$ and $B_{i}$, such that

$$
\begin{aligned}
\sigma\left(\sigma_{j}(\underline{a}) \cdot \underline{b}\right) & =\sigma\left(\sigma_{j}\left(\underline{a}_{0}\right) \cdot \underline{b}_{0}\right), \\
\sigma_{r}\left(\sigma_{1}(\underline{a}) \cdot \underline{b}\right) & =\sigma_{r}\left(\sigma_{1}\left(\underline{a}_{0}\right) \cdot \underline{b}_{0}\right)
\end{aligned}
$$

and

$$
\sigma_{1}\left(\sigma_{r}(\underline{a}) \cdot \underline{b}\right)=\sigma_{1}\left(\sigma_{r}\left(\underline{a}_{0}\right) \cdot \underline{b}_{0}\right)
$$

where $\underline{a}_{0}=\left(\pi_{0}\left(A_{1} \mid \mathscr{H}_{0}\right), \ldots, \pi_{0}\left(A_{n} \mid \mathscr{H}_{0}\right)\right)$, and $\pi_{0}$ is the quotient projection from $\mathcal{L}\left(\mathscr{H}_{0}\right)$ onto $\mathcal{L}\left(\mathscr{H}_{0}\right) / \mathcal{K}\left(\mathscr{H}_{0}\right)$.

If $P_{0}$ is the orthogonal projection from $\sharp$ onto $\mathscr{H}_{0}$, then the elementary multiplication on $A$, denoted by $S_{0}$, which maps $x \in A$ into $\pi\left(P_{0}\right) x \pi\left(P_{0}\right)$ is in fact a projection, and its range is a reducing subspace for $L_{\underline{a}} \cdot R_{\underline{b}}$, thus defining the restriction $L_{\underline{a}_{0}} \cdot R_{\underline{a}_{0}}=\left(L_{\underline{a}} \cdot R_{\underline{b}}\right) \mid \operatorname{Im} S_{0}$. By Theorem 1, (1.20)-(1.22), and the already proved (1.19) for the separable case, it follows that

$$
\begin{aligned}
\sigma\left(L_{\underline{a}} \cdot R_{\underline{b}}\right) & =\sigma_{r}\left(\sigma_{1}(\underline{a}) \cdot \underline{b}\right) \cup \sigma_{1}\left(\sigma_{r}(\underline{a}) \cdot \underline{b}\right) \\
& =\sigma_{r}\left(\sigma_{1}\left(\underline{a}_{0}\right) \cdot \underline{b}_{0}\right) \cup \sigma_{1}\left(\sigma_{r}\left(\underline{a}_{0}\right) \cdot \underline{b}_{0}\right) \\
& =\sigma\left(L_{\underline{a}_{0}} \cdot R_{\underline{b}_{0}}\right)=\sigma\left(\sigma_{j}\left(\underline{a}_{0}\right) \cdot \underline{b}_{0}\right)=\sigma\left(\sigma_{j}(\underline{a}) \cdot \underline{b}\right) .
\end{aligned}
$$

COROLlARY 2. If the $n$-tuples $\underline{a}$ and $\underline{b}$ of elements in $A^{n}$ are both commutative, then

$$
\sigma\left(L_{\underline{a}} \cdot R_{\underline{b}}\right)=\sigma_{j}(\underline{a}) \cdot \sigma_{j}(\underline{b}) .
$$

PROOF. This a consequence of $(0.6)$ and Theorem 3.

We conclude with a generalization of a result of L. A. Fialkow [5, Theorem 2.5].

COROLLARY 3. An elementary operator $L_{\underline{A}} \cdot R_{\underline{B}}$ on $\mathcal{L}(\mathcal{H})$, where the $n$-tuple $\underline{a}=\pi(\underline{A})$ (respectively, $\underline{b}=\pi(\underline{B})$ ) is commutative, has dense range if and only if $\lambda \notin \sigma_{1}\left(\sigma_{r}(\underline{a}) \cdot \underline{b}\right)$ (respectively, $\lambda \notin \sigma_{r}\left(\sigma_{1}(\underline{b}) \cdot \underline{a}\right)$ ) and there does not exist a nonzero trace-class operator $T$ such that $\left(L_{\underline{B}} \cdot R_{\underline{A}}-\lambda\right) T=0$.

ProOF. By [15, Theorem 2] $L_{\underline{A}} \cdot R_{\underline{B}}-\lambda$ has dense range if and only if the restriction $\left(L_{\underline{A}} \cdot R_{\underline{B}}-\lambda\right) \mid \mathcal{K}(\not{K})$ and $L_{\underline{a}} \cdot R_{\underline{b}}-\lambda \in \mathcal{L}(\mathcal{A})$ both have dense range. But $\left(L_{\underline{A}} \cdot R_{\underline{B}}-\lambda\right) \overline{\mid K}(\not H)$ has dense range if and only if its Banach space adjoint $\left(L_{\underline{B}} \cdot R_{\underline{A}}-\bar{\lambda} \mid C_{1}(\not h)\right.$ is injective and $L_{\underline{a}} \cdot R_{\underline{b}}-\lambda$ has dense range if and only if $\lambda \notin \sigma_{1}\left(\sigma_{r}(\underline{a}) \cdot \underline{b}\right)$ (respectively, $\lambda \notin \sigma_{r}\left(\sigma_{1}(\underline{b}) \cdot \underline{a}\right)$ ).

ACKNOWLEDGEMENT. The author owes gratitude to B. Magajna for a clue. $\mathrm{He}$ is also thankful to Professor M. Omladic for encouragement, to Professor L. A. Fialkow for a preprint of $[4]$ and to the referee for his great care and helpful advice. 


\section{REFERENCES}

1. W. Arveson, Notes on extensions of $C^{*}$-algebras, Duke Math. J. 44 (1977), 329-355.

2. A. Carrillo and C. Hernandez, Spectra of constructs of a system of operators, Proc. Amer. Math. Soc. 91 (1984), 426-432.

3. A. T. Dash, Joint essential spectra, Pacific J. Math. 64 (1976), 119-128.

4. L. A. Fialkow, $A$ note on the operator $X \rightarrow A X-A B$, Trans. Amer. Math. Soc. 243 (1978), 147-168.

5. __ Spectral properties of elementary operators, Acta Sci. Math. (Szeged) 46 (1983), 269-282.

6. _ Spectral properties of elementary operators.II, (preprint).

7. P. A. Fillmore, J. G. Stampfli and J. P. Williams, On the essential numerical range, esssential spectrum and a problem of Halmos, Acta Sci. Math. (Szeged) 33 (1972), 179192.

8. C. K. Fong and A. R. Sorour, On the operator identity $\sum A_{k} X B_{k} \equiv 0$, Canad. J. Math. 31 (1979), 845-857.

9. R. E. Harte, Spectral mapping theorems, Proc. Roy. Irish Acad. Sect. A 72 (1972), 89-107.

10. __ Spectral mapping theorems for quasicommuting systems, Proc. Roy. Irish Acad. Sect. A 73 (1973), 7-18.

11. __ Tensor products, multiplication operators and the spectral mapping theorems, Proc. Roy. Irish Acad. Sect. A 73 (1973), 285-302.

12. G. Lumer and M. Rosenblum, Linear operator equations, Proc. Amer. Math. Soc. 10 (1959), 32-41.

13. M. Mathieu, Spectral theory for multiplication operators on $C^{*}$-algebras, Proc. Roy. Irish Acad. Sect. A 83 (1983), 231-249.

14. D. Voiculescu, A non-commutative Weyl-von Neumann theorem, Rev. Roumaine Math. Pures Appl. 21 (1976), 97-113.

15. R. E. Weber, On weak*-continuous operators on $\mathcal{L}(\mathscr{H})$, Proc. Amer. Math. Soc. 83 (1981), 735-742.

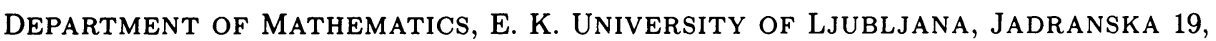
61000 LJUBLJANA, YUgOSLAVIA 